\title{
Dihedral f-tilings of the sphere by rhombi and triangles
}

\author{
Ana M. Breda1 $\|^{\dagger}$ and Altino F. Santos $2 ⿰$ \\ ${ }^{1}$ Department of Mathematics, University of Aveiro, 3810 - 193 Aveiro, Portugal \\ e-mail: ambreda@mat.ua.pt \\ ${ }^{2}$ Department of Mathematics, U.T.A.D., 5001 - 911 Vila Real, Portugal \\ e-mail: afolgado@utad.pt \\ received Mar 19, 2004, revised Jan 28, 2005, accepted May 31, 2005.
}

We classify, up to an isomorphism, the class of all dihedral f-tilings of $S^{2}$, whose prototiles are a spherical triangle and a spherical rhombus. The equiangular case was considered and classified in Breda and Santos (2004). Here we complete the classification considering the case of non-equiangular rhombi, see Tab. 1] and Fig. 24.

Keywords: dihedral tilings, isometric foldings, spherical trigonometry, WCSQ

\section{Introduction}

An isometric folding is a non-expansive locally isometry that sends piecewise geodesic segments into piecewise geodesic segments of the same length. An isometric folding is a continuous map that need not to be differentiable. The points where it is not differentiable are called singular points. The foundations of isometric foldings of Riemannian manifolds are introduced by Robertson (1977).

For surfaces, the set of singular points gives rise to two colored tilings (called f-tilings) with the property that each vertex has even valency and obey the angle folding relation, i.e., the sums of alternating angles around each vertex is $\pi$.

The classification of f-tilings started out by Breda (1992), where a complete classification of all monohedral f-tilings of the sphere by triangles was done.

The complete classification of monohedral tilings of the sphere by triangles (which, obviously, includes the monohedral triangular f-tilings) was made clear by Ueno and Agaoka (2002). This classification was partially done by Sommerville (1922), and an outline of the proof was provided by Davies (1967).

For additional information on tilings, see Grünbaum and Shephard (1986).

Our interest focuses in dihedral spherical f-tilings.

\footnotetext{
${ }^{\dagger}$ Supported in part by UI\&D Matemática e Aplicações of University of Aveiro, through Program POCTI of FCT cofinanced by the European Community fund FEDER.

¥Supported in part by UI\&D Matemática e Aplicações of University of Aveiro, through Program POCTI of FCT cofinanced by the European Community fund FEDER.

1365-8050 @ 2005 Discrete Mathematics and Theoretical Computer Science (DMTCS), Nancy, France
} 


\section{Preliminaries}

Definition 2.1 Let $S^{2}$ be the euclidean 2-sphere of radius 1. A spherical moon $L$ is said well centered if its vertices belong to the great circle $S^{2} \cap\left\{(x, y, z) \in \mathbb{R}^{3} \mid x=0\right\}$ and the semi-great circle bisecting $L$ contains the point $(1,0,0)$. If $L_{1}$ and $L_{2}$ are two well centered spherical moons with orthogonal vertices, then $L_{1}$ and $L_{2}$ are said to be orthogonal.

Definition 2.2 By a well centered spherical quadrangle (WCSQ) we mean a spherical quadrangle which is the intersection of two well centered spherical moons with distinct vertices.

The properties of WCSQ was described by Breda and Santos (2003), where was established that any spherical quadrangle with congruent opposite internal angles is congruent to a WCSQ.

Definition 2.3 Let $Q$ and $T$ be, respectively, a spherical triangle and a spherical quadrangle. A dihedral tiling of the sphere $S^{2}$ with prototiles $Q$ and $T$ is an edge-to-edge polygonal subdivision $\tau$ of $S^{2}$, such that each tile (face) of $\tau$ is isometric either to $Q$ or $T$.

In addition, if the number of geodesic rays (edges) emanating from each vertex of $\tau$ is even, making alternated angles $\alpha_{1}, \beta_{1}, \alpha_{2}, \beta_{2}, \ldots, \alpha_{n}, \beta_{n}(n \geq 2)$, where

$$
\sum_{j=1}^{n} \alpha_{j}=\sum_{j=1}^{n} \beta_{j}=\pi \quad \text { (angle folding relation) }
$$

then $\tau$ is called a dihedral folding tiling or dihedral f-tiling for short.

In short, dihedral means that every tile of $\tau$ is congruent to one of the fixed sets $Q$ and $T$, called the prototiles of $\tau$.

Definition 2.4 Let $v$ be a vertex of a (dihedral) f-tiling. The number of edges emanating from $v$ is called the valency of $v$.

In Fig. 1 is illustrated a vertex $v$ obeying the angle folding relation $\left(\alpha_{1}+\alpha_{2}+\alpha_{3}=\beta_{1}+\beta_{2}+\beta_{3}=\pi\right)$ with valency six.

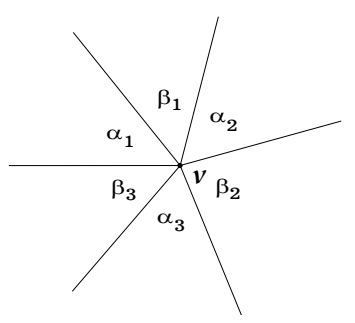

Fig. 1: The angle folding relation.

In Breda and Santos (2004) the classification of dihedral f-tilings of $S^{2}$ by spherical triangles and equiangular spherical quadrangles (i.e., spherical quadrangles that are congruent to a WCSQ which is the intersection of two orthogonal well centered spherical moons) was presented. 
Here we extend the study of f-tilings presenting the classification of all dihedral f-tilings by spherical triangles and non-equiangular spherical rhombi, in other words, spherical quadrangles whose edges have the same length and with distinct pairs of opposite internal angles.

In Breda and Santos (2003) it was shown that any non-equiangular spherical rhombus $Q$ is congruent to a WCSQ which is the intersection of two non-orthogonal well centered spherical moons $L_{1}$ and $L_{2}$ with the same angle measure $\theta$ (Fig.2).

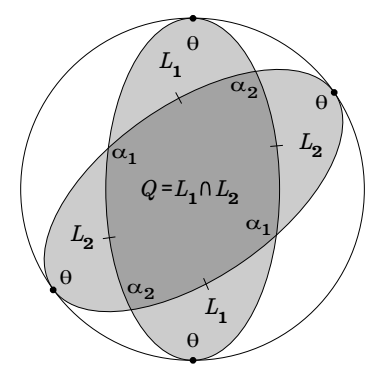

Fig. 2: A non-equiangular spherical rhombus $Q$.

Definition 2.5 The spherical tilings $\tau_{1}$ and $\tau_{2}$ are isomorphic if there exists an isometry $\phi$ of $S^{2}$, such that $\phi\left(\tau_{1}\right)=\tau_{2}$.

Notation: We shall denote by $\Omega(Q, T)$ the set, up to an isomorphism, of all dihedral f-tilings of $S^{2}$, whose prototiles are a non-equiangular spherical rhombus $Q$ and a spherical triangle $T$.

Proposition 2.1 (Breda and Santos (2004)) Let $\tau \in \Omega(Q, T)$. If $M>0$ and $N>0$ denote, respectively, the number of spherical quadrangles congruent to $Q$ and the number of spherical triangles congruent to $T$ of $\tau$, and $E$ and $V$ denote, respectively, the number of edges and vertices of $\tau$, then:

i) $N+2 M=2 V-4,3 V=6+M+E$;

ii) there are at least $6+M$ vertices of valency four;

iii) $\pi<\beta+\gamma+\delta<\frac{3 \pi}{2}$ and $\alpha_{1}+\alpha_{2}>\pi$, where $\alpha_{1}$ and $\alpha_{2}$ are the pairs of opposite internal angles of $Q$, and $\beta, \gamma$ and $\delta$ are the internal angles of $T$.

We shall describe $\Omega(Q, T)$, considering separately different cases depending on the nature of $Q$ and $T$. In order to get the dihedral f-tilings of $\Omega(Q, T)$ we find useful to start by considering one of its planar representation $(P R)$, beginning with a common vertex to a spherical quadrangle and a spherical triangle in adjacent positions (it must be pointed out that there is always a vertex satisfying this condition).

Whenever a tiling is drown in one hemisphere, it means that the plane $x=0$, say, is a plane of symmetry. The whole tiling is then obtained by reflecting the picture in this plane.

Spherical trigonometry is based on the following cosine rules: The internal angles $\alpha, \beta$ and $\gamma$ of a spherical triangle $T$ obey:

$$
\cos \alpha=\frac{\cos a-\cos b \cos c}{\sin b \sin c} \text { and } \cos a=\frac{\cos \alpha+\cos \beta \cos \gamma}{\sin \beta \sin \gamma}
$$


where $a, b$ and $c$ are the lengths of the edges opposite to $\alpha, \beta$ and $\gamma$, respectively. This formulas are of crucial interest to prove the results that follow. For a detailed discussion on spherical trigonometry, see Berger (1996).

\section{Prototiles: Non-equiangular rhombus and equilateral triangle}

Proposition 3.1 If $Q$ and $T$ are, respectively, a non-equiangular spherical rhombus and an equilateral spherical triangle, then $\Omega(Q, T)$ is the empty set.

Proof: Let $Q$ be a non-equiangular spherical rhombus with pairs of opposite internal angles $\alpha_{1}$ and $\alpha_{2}$ $\left(\alpha_{1}>\alpha_{2}\right)$, and let $T$ be an equilateral spherical triangle with internal angle $\beta$.

Suppose that $\Omega(Q, T)$ is non empty. Let $v$ be a vertex of $Q$ surrounded by $\alpha_{1}$. As $\alpha_{1}+\alpha_{2}>\pi$ $\left(\alpha_{1}>\frac{\pi}{2}\right)$ and $\beta>\frac{\pi}{3}$, then $v$ has valency four, and the cells surrounding $v$ have, in cyclic order, angles measure $\left(\alpha_{1}, \alpha_{1}, \beta, \beta\right)$, with $\alpha_{1}+\beta=\pi$. A planar representation (PR) of a f-tiling $\tau \in \Omega(Q, T)$ near $v$ is illustrated in Fig. 3-I. It follows that $\alpha_{2}+\beta<\pi$ and $\alpha_{2}+k \beta>(k+1) \beta>\pi$, for $k \geq 2$. Hence in order to have the angle folding relation satisfied we must have $\alpha_{2}+\alpha_{2}=\pi$, i.e., $\alpha_{2}=\frac{\pi}{2}$. This information allows us to adjust some more cells to the initial $P R$ leading to the $P R$ illustrated in Fig. 3-II. In order to go on with the $P R$ of $\tau$ we must analyze the angles behavior around $v_{1}$. We already know that the cyclic sequence of their positions contains the sequence $\left(\ldots, \beta, \alpha_{2}, \beta, \ldots\right)$. As $2 \beta<\pi$ and $2 \beta+\rho>\pi$ for all $\rho \in\left\{\beta, \alpha_{1}, \alpha_{2}\right\}$, then there is no way to position another cells around $v_{1}$ in order to have the angle folding relation full filled. And so $\Omega(Q, T)=\emptyset$.

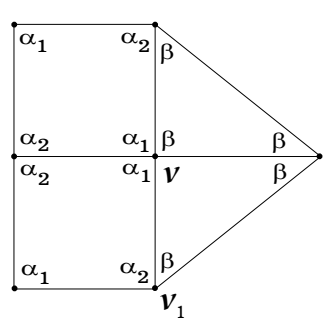

I

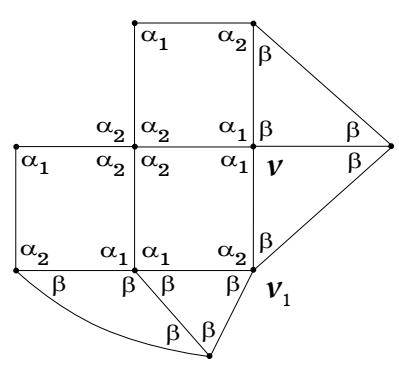

II

Fig. 3: Planar representations.

The argumentation used to prove the Propositions stated

in the following Sections are somehow similar to the one used on proof of Prop. 3.1. Thus, we decided to present only the proofs we consider more representative. A detailed study containing the proofs of those results can be found in a technical report placed in the web site

http://www.mat.ua.pt/ambreda/rhombi.pdf 


\section{Prototiles: Non-equiangular rhombus and isosceles triangle}

Through this section $Q$ is a non-equiangular spherical rhombus with pairs of opposite internal angles $\alpha_{1}$ and $\alpha_{2}\left(\alpha_{1}>\alpha_{2}\right)$, and $T$ is an isosceles spherical triangle with internal angles $\beta, \gamma, \gamma(\beta \neq \gamma)$.

Any element of $\Omega(Q, T)$ has, at least, two cells congruent, respectively, to $Q$ and $T$, such that they are in adjacent positions in one of the situations illustrated in Fig. 4

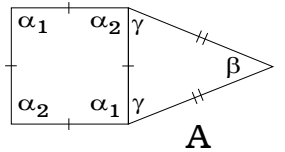

A

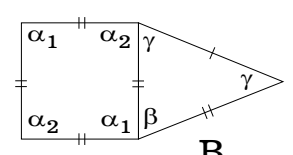

B

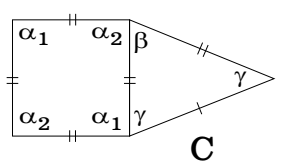

C

Fig. 4: Distinct cases of adjacency.

Next, by "unique f-tiling" we mean unique up to an isomorphism.

Proposition 4.1 If $\Omega(Q, T) \neq \emptyset$, then there cannot be a pair of tiles in the position of Fig. 4.A or Fig. 4 $B$.

Proposition 4.2 If a f-tiling contains two cells in the position of Fig. 4. C, then $\alpha_{1}+\gamma=\pi$. Moreover,

i) if $\alpha_{2} \neq \beta$, then $\Omega(Q, T) \neq \emptyset$ iff $\alpha_{2}+\beta=\pi\left(\beta=\arccos \left(1-2 \cos \frac{\pi}{k}\right)\right)$ and $\gamma=\frac{\pi}{k}$, for some $k \geq 4$. In this situation for each $k \geq 4, \Omega(Q, T)$ contains a unique dihedral $f$-tiling, denoted by $R^{k}$, such that the angles around vertices are the ones represented at the head of the Fig.7 (consider $k \geq 4$ );

ii) if $\alpha_{2}=\beta$, then $\Omega(Q, T) \neq \emptyset$ iff $\alpha_{1}=\frac{2 \pi}{3}, \alpha_{2}=\frac{\pi}{2}$ and $\gamma=\frac{\pi}{3}$. Besides, $\Omega(Q, T)=\left\{R^{3}, U_{1}, U_{2}, U_{3}, U_{4}\right\}$, where $R^{3}$ and $U_{i}, 1 \leq i \leq 4$ are non-isomorphic dihedral $f$-tilings, such that the angles around vertices are the ones represented in Fig. 7

Proof: We only present the proof of the ii) case.

In order to obtain a complete $P R$ of an element $\tau \in \Omega(Q, T)$, the $P R$ illustrated in Fig. 4. C is extended in a unique way to get the $P R$ illustrated in Fig. 5, where $\alpha_{1}+\gamma=\pi, \alpha_{2}+\beta=\pi$ and $k \gamma=\pi$, for some $k \geq 4$. (Note that the case $k=3$ implies $\alpha_{2}=\beta=\frac{\pi}{2}$.)

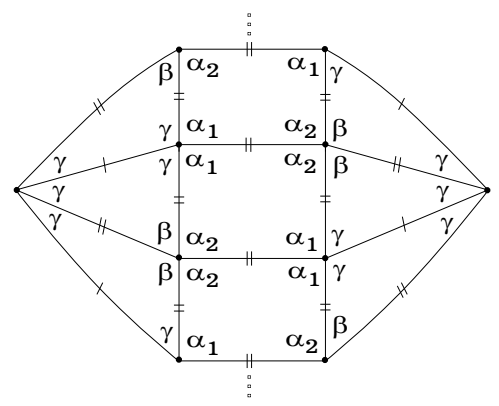

Fig. 5: $P R$ of $R^{k}, k \geq 4$. 
The side length of $T$ opposite to $\gamma$ and the side length of $Q$ are the same. Using the formulas 2.1 we may show that

$$
\beta=\arccos \left(1-2 \cos \frac{\pi}{k}\right)=2 \arccos \sqrt{1-\cos \frac{\pi}{k}}, k \geq 4
$$

3D representations for $k=4$ and $k=5$ are illustrated in Fig. 6
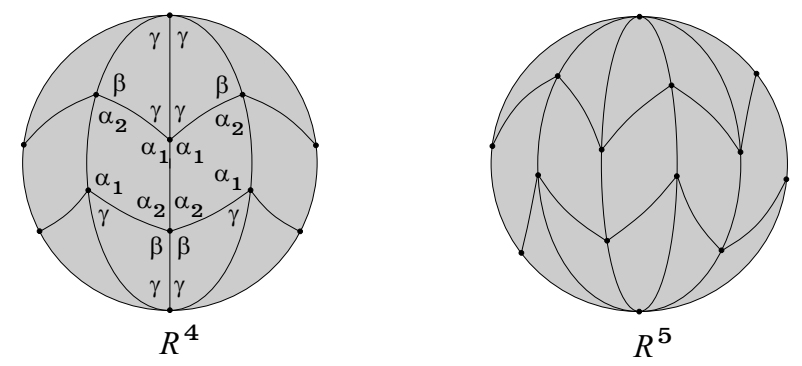

Fig. 6: 3D representations.

Fig. 7 exhibits the vertices in the dihedral f-tilings corresponding to the ii and ii) cases. In Fig. 8

$$
\begin{aligned}
& \begin{array}{c|cc|cc}
\alpha_{1} & \alpha_{1} \\
\hline \gamma & \gamma & \alpha_{2} & \alpha_{2} \\
\hline \beta & \beta & \begin{array}{c}
\gamma \gamma \gamma / \gamma \\
\gamma / \gamma \\
k \gamma=\pi
\end{array}
\end{array} \quad R, k \geq 3
\end{aligned}
$$

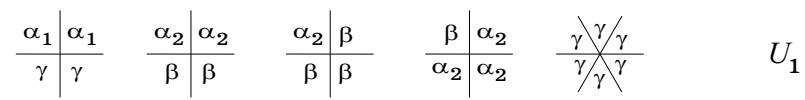

$$
\begin{aligned}
& \begin{array}{r|r|rr|rr}
\alpha_{1} & \alpha_{1} \\
\hline \gamma & \gamma & \beta & \alpha_{2} & \alpha_{2} & \beta \\
\hline \alpha_{2} & \alpha_{2} & \beta & \alpha_{2} & U_{2}
\end{array}
\end{aligned}
$$

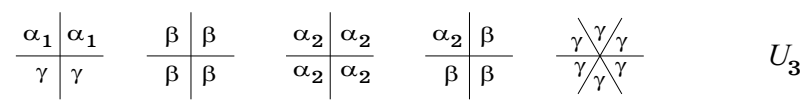

$$
\begin{aligned}
& \begin{array}{c|cc|cc|cc}
\alpha_{1} & \alpha_{1} \\
\hline \gamma & \gamma & \frac{\alpha_{2}}{\alpha_{2}} & \alpha_{2} & \alpha_{2} & \beta \\
\hline \alpha_{2} & \alpha_{2} & \alpha_{2} & U_{4}
\end{array}
\end{aligned}
$$

Fig. 7: Distinct classes of congruent vertices.

are illustrated 3D representations of the f-tilings refereed in ii) case. For additional information on these tilings, see Tab. 1 . 

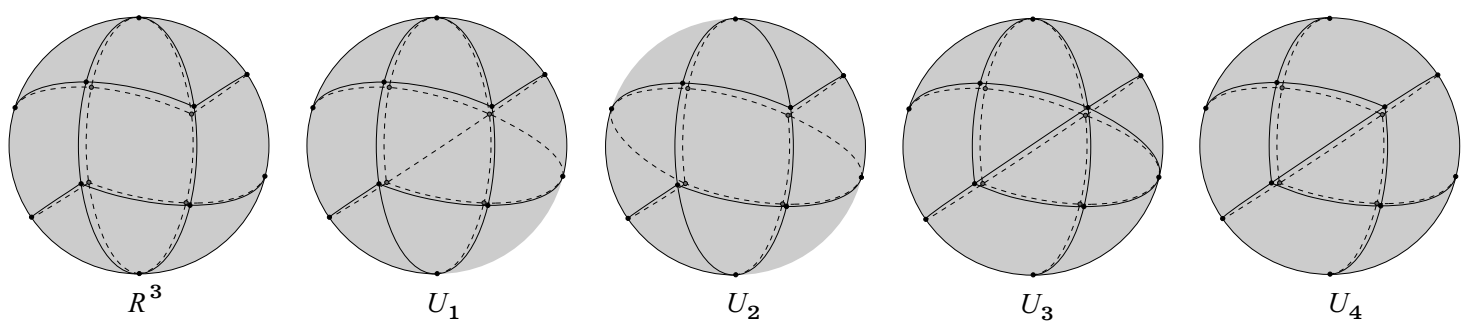

Fig. 8: 3D representations.

\section{Prototiles: Non-equiangular rhombus and scalene triangle}

Here $Q$ is a non-equiangular spherical rhombus with pairs of opposite internal angles $\alpha_{1}$ and $\alpha_{2}\left(\alpha_{1}>\right.$ $\alpha_{2}$ ), and $T$ is a scalene spherical triangle with internal angles $\beta, \gamma$ and $\delta$, such that $\beta>\gamma>\delta$.

If $\tau \in \Omega(Q, T)$, then there are necessarily two cells of $\tau$ congruent, respectively, to $Q$ and $T$, such that they are in adjacent positions in one of the situations illustrated in Fig. 9
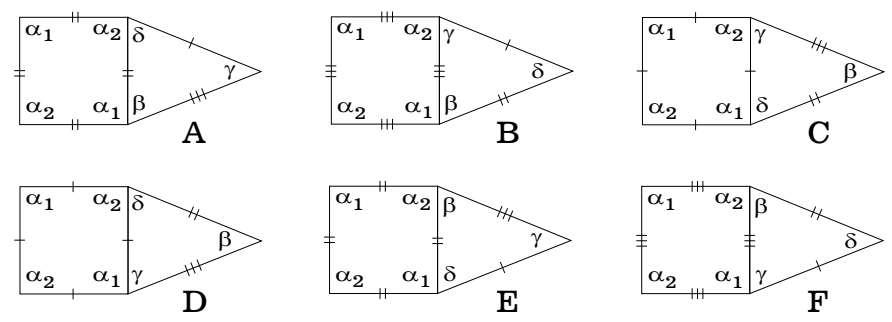

Fig. 9: Distinct cases of adjacency.

Proposition 5.1 If $\Omega(Q, T) \neq \emptyset$, then there cannot be a pair of tiles in the position of Fig. 아A or Fig. 98 $B$.

Proposition 5.2 Let $\tau \in \Omega(Q, T)$, such that $\tau$ has two cells in adjacent positions as illustrated in Fig. 9 . $C$, then $\tau$ has six cells in adjacent positions as illustrated in Fig. 10.

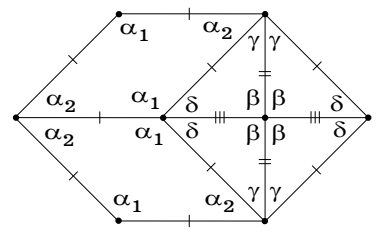

Fig. 10: Planar representation.

Suppose that the $P R$ illustrated in Fig. 10 is contained in a complete $P R$ of any element $\tau \in \Omega(Q, T)$. It follows that $\alpha_{2}+\gamma \leq \pi$. We shall consider separately the cases $\alpha_{2}+\gamma<\pi$ and $\alpha_{2}+\gamma=\pi$. 
Proposition 5.3 Let $\alpha_{2}+\gamma<\pi$.

i) If $\alpha_{2}>\gamma$, then $\tau=M^{4}$;

ii) If $\alpha_{2}=\gamma$, then $\tau=E^{3}$ or $\tau=S^{3}$;

iii) If $\alpha_{2}<\gamma$, then $\tau=M^{k+1}$ or $\tau=E^{k}$ or $\tau=S^{k}, k \geq 4$,

where the internal angles of the prototiles present in the dihedral f-tilings are:

$$
\begin{array}{lrrrrrl}
\alpha_{1}=\frac{(k-1) \pi}{k} & \alpha_{2}=\pi-2 \gamma & \beta=\frac{\pi}{2} & \cos \gamma=\sqrt{1-\cos \frac{\pi}{k}} & \delta=\frac{\pi}{k} & M^{k}, k \geq 4 ; \\
\alpha_{1}=\pi-\delta & \alpha_{2}=\frac{\pi}{2 k-3} & \beta=\frac{\pi}{2} & \gamma=\frac{(k-2) \pi}{2 k-3} & \cos \delta=\cos ^{2} \frac{\pi}{4 k-6} & E^{k}, k \geq 3 ; \\
\alpha_{1}=\pi-\delta & \alpha_{2}=\frac{\pi}{k} & \beta=\frac{\pi}{2} & \gamma=\frac{(k-1) \pi}{2 k} & \cos \delta=\cos ^{2} \frac{\pi}{2 k} & S^{k}, k \geq 3 .
\end{array}
$$

The angles around vertices are positioned as illustrated in Fig. 11

$$
\begin{aligned}
& \begin{array}{l|l}
\alpha_{1} & \alpha_{1} \\
\hline \delta & \delta
\end{array} \\
& \begin{array}{l|l}
\beta & \beta \\
\hline \beta & \beta
\end{array} \\
& \frac{\alpha_{2} \alpha_{2} / \gamma}{\gamma / \gamma} \\
& \begin{array}{l}
\delta / \delta / \delta \\
\delta / \delta \\
k \delta=\pi
\end{array} \\
& \begin{array}{l|l}
\alpha_{1} & \alpha_{1} \\
\hline \delta & \delta
\end{array} \\
& \begin{array}{l|l}
\beta & \beta \\
\hline \beta & \beta
\end{array} \\
& \frac{\gamma / \alpha_{2} / \gamma}{\gamma / \alpha_{2} \gamma} \\
& \left.\therefore \gamma_{\alpha_{2}}^{\alpha_{2}}\right|_{\alpha_{2}} ^{\alpha_{2}} \gamma \\
& (k-1) \alpha_{2}+\gamma=\pi \\
& \begin{array}{l|l}
\alpha_{1} & \alpha_{1} \\
\hline \delta & \delta
\end{array} \\
& \begin{array}{l|l}
\beta & \beta \\
\hline \beta & \beta
\end{array} \\
& \frac{\gamma / \alpha_{2} / \gamma}{\gamma / \alpha_{2} \gamma} \\
& \therefore \alpha_{\alpha_{2}}^{\alpha_{2}} \alpha_{\alpha_{2}}^{\alpha_{2}} \alpha_{2} \alpha_{2} \\
& M^{k}, k \geq 4 \\
& E^{k}, k \geq 3 \\
& S^{k}, k \geq 3
\end{aligned}
$$

Fig. 11: Distinct classes of congruent vertices.

Proof: We only present the proof of the ii) case.

The information given by the $P R$ illustrated in Fig. 10 leads to conclude that

$$
\alpha_{1}>\beta=\frac{\pi}{2}>\alpha_{2}>\gamma>\delta .
$$

Consider a vertex $v$ surrounded by the cyclic sequence $\left(\alpha_{2}, \gamma, \gamma, \ldots\right)$. Then the sum of alternating angles around $v$ containing $\alpha_{2}$ must be $\alpha_{2}+\gamma+\alpha_{2}=\pi$ or $\alpha_{2}+2 \gamma=\pi$.

1. $\alpha_{2}+\gamma+\alpha_{2}=\pi$

In this case the $P R$ illustrated in Fig. 10 is extended as shown in Fig. 12 (observe that all cells labelled from 2 to 8 are uniquely determined, since $\alpha_{2}+\gamma<\alpha_{2}+2 \gamma<\pi=2 \alpha_{2}+\gamma<\alpha_{2}+\gamma+2 \delta$ ). This leads to a 


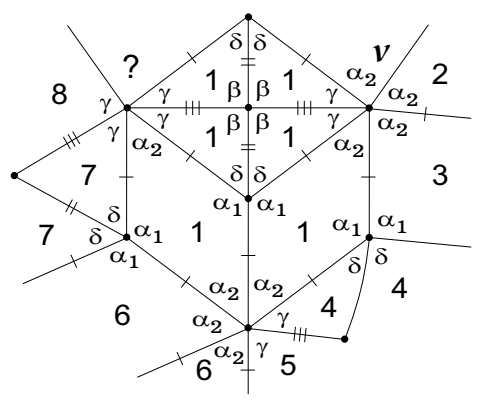

Fig. 12: Planar representation.

vertex surrounded by the cyclic sequence of angles $\left(\gamma, \gamma, \alpha_{2}, \gamma, \gamma, \ldots\right)$, where $2 \gamma+\alpha_{2}<\pi<2 \gamma+\alpha_{2}+\rho$, for all $\rho \in\left\{\alpha_{1}, \alpha_{2}, \beta, \gamma, \delta\right\}$. And so there is no way to obey the angle folding relation at that vertex.

2. $\alpha_{2}+2 \gamma=\pi$

In this case the cyclic sequence of angles around $v$ is either $\left(\alpha_{2}, \gamma, \gamma, \alpha_{2}, \gamma, \gamma\right)$ or $\left(\alpha_{2}, \gamma, \gamma, \gamma, \gamma, \alpha_{2}\right)$.

2.1. The first situation leads us immediately to a contradiction as illustrates Fig. 13 In fact, this case gives rise to a vertex $w$ surrounded by the cyclic sequence $\left(\alpha_{2}, \alpha_{2}, \alpha_{2}, \alpha_{2}, \ldots\right)$. As $\alpha_{2}>\gamma$, then $2 \alpha_{2}+\gamma>\pi=\alpha_{2}+2 \gamma>2 \alpha_{2}$. And so the sums of alternating angles around $w$ must obey $2 \alpha_{2}+\delta=\pi$, as illustrated in the figure. This procedure gives rise to a vertex surrounded by $\alpha_{1}$ and $\gamma$ in adjacent positions, leading us to an incompatibility between angles around this vertex (see side length of the prototiles).

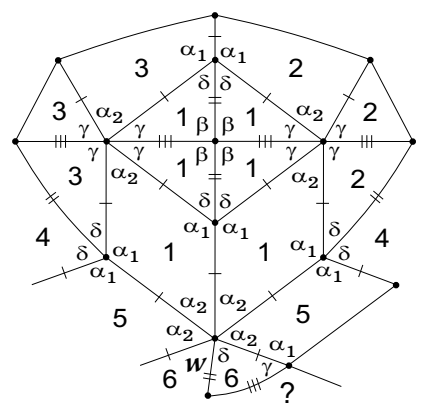

Fig. 13: Planar representation.

2.2. In the second case we may extend without more restrictions the $P R$ illustrated in Fig. 10 to get the one illustrated in Fig. 14. This construction leads to two antipodal vertices surrounded by at least six $\delta$ angles. It is a straightforward exercise to show that those vertices are enclosed exclusively by $\delta$ angles. Therefore $k \delta=\pi$, for some $k \geq 4$. On the other hand $\alpha_{2}+2 \gamma=\pi$ with $\gamma<\alpha_{2}$. Hence $\gamma<\frac{\pi}{3}<\alpha_{2}$ and so $\delta=\frac{\pi}{4}$ or $\delta=\frac{\pi}{5}$, as $\gamma+\delta>\frac{\pi}{2}$.

Firstly, consider that $\delta=\frac{\pi}{4}$. Then $\alpha_{1}=\frac{3 \pi}{4}$ and the $P R$ illustrated in Fig. 14 is completely determined 


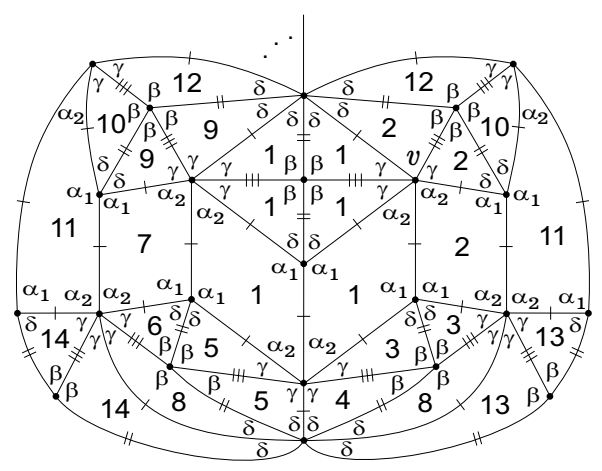

Fig. 14: Planar representation.

as shown in Fig. 15. The sum of alternating angles around vertices verify

$$
\alpha_{1}+\delta=\pi, \quad \beta=\frac{\pi}{2}, \quad \alpha_{2}+2 \gamma=\pi\left(\alpha_{2}>\gamma\right) \quad \text { and } \quad \delta=\frac{\pi}{4}
$$

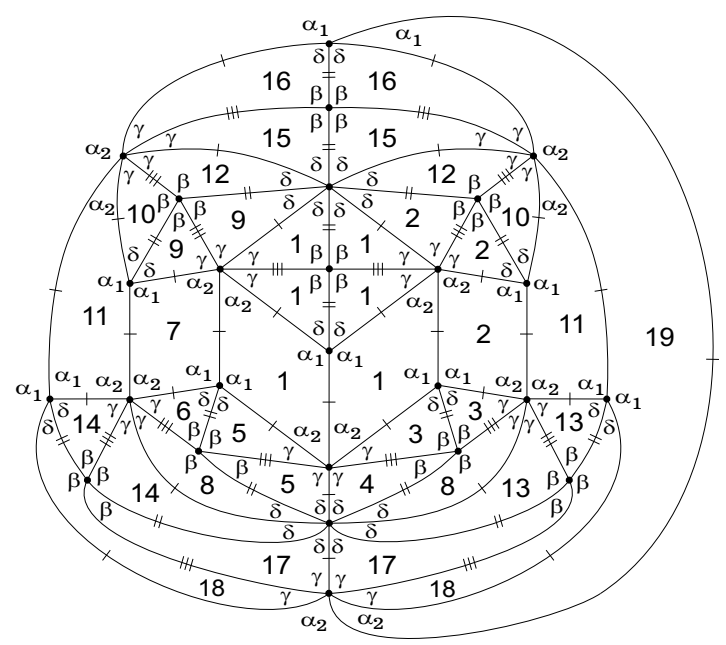

Fig. 15: Extended planar representation.

Now, we need to verify that adjacent tiles have the same side length. Denoting by $b$ the side length of $T$ opposite to $\beta$, then

$$
\cos b=\frac{\cos \beta+\cos \delta \cos \gamma}{\sin \delta \sin \gamma}=\cot \gamma
$$


On the other hand $b$ is also the side length of $Q$, therefore

$$
\cos b=\frac{\cos \frac{\alpha_{2}}{2}\left(1+\cos \alpha_{1}\right)}{\sin \frac{\alpha_{2}}{2} \sin \alpha_{1}}=\frac{\cos \left(\frac{\pi}{2}-\gamma\right)\left(1+\cos \alpha_{1}\right)}{\sin \left(\frac{\pi}{2}-\gamma\right) \sin \alpha_{1}}=\tan \gamma \frac{1-\cos \frac{\pi}{4}}{\sin \frac{\pi}{4}}
$$

By (5.2) and (5.3) we conclude that

$$
\tan ^{2} \gamma=\frac{\cos \frac{\pi}{4}}{1-\cos \frac{\pi}{4}}
$$

And so

$$
\gamma=\arccos \sqrt{1-\cos \frac{\pi}{4}}=\frac{1}{2} \arccos (1-\sqrt{2}) \approx 57^{\circ} .
$$

To complete the proof we shall consider now that $\delta=\frac{\pi}{5}$ (Fig. 14). As before the side length of $T$, $b$, opposite to $\beta$ is common to $Q$, leading to conclude that $\gamma=\arccos \sqrt{1-\cos \frac{\pi}{5}}=\arccos \frac{\sqrt{3-\sqrt{5}}}{2} \approx$ $64^{\circ}>\frac{\pi}{3}$, contradicting 5.1 .

3D representations of some of these f-tilings are illustrated in Fig. 16 . For additional information see also Tab. 1 .
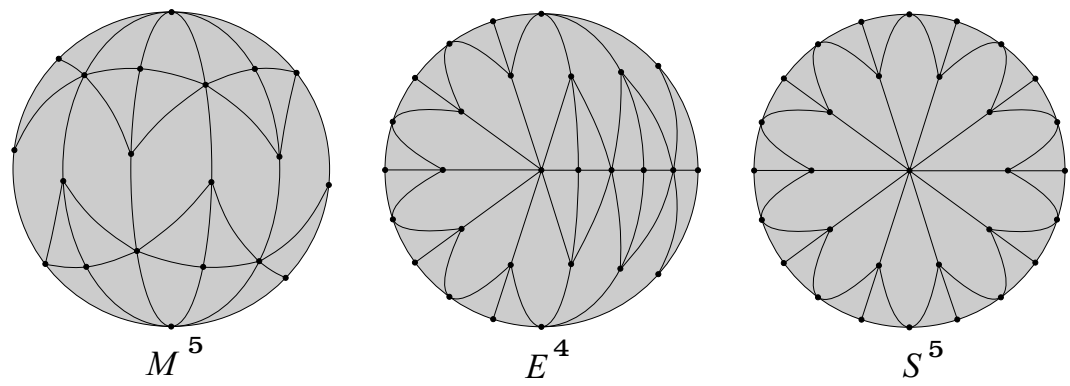

Fig. 16: 3D representations.

Remark: A 3D representation of the dihedral f-tilings $M^{k}, k \geq 4$ is obtained from a 3D representation of $R^{k}, k \geq 4$ (Prop. 4.2 i) ) by adding some edges bisecting by $\beta=2 \arccos \sqrt{1-\cos \frac{\pi}{k}}$ any isosceles triangle of $R^{k}$ and preserving the angle-folding relation. Compare, for instance, the f-tilings $M^{5}$ (above, Fig. 16, and $R^{5}$ (Fig. 6).

Proposition 5.4 If $\alpha_{2}+\gamma=\pi$, then $\Omega(Q, T)$ is the family of f-tilings denoted by $\left(R_{\delta}^{2}\right)_{\delta \in] 0, \frac{\pi}{3}}$, where $\cos \gamma+\cos \delta=1$. (Its $3 D$ representation is illustrated in Fig. 17)

Remark: If $\delta=\frac{\pi}{3}$, then $\gamma=\frac{\pi}{3}$ and so $\alpha_{1}=\alpha_{2}=\frac{2 \pi}{3}$, giving rise to a dihedral f-tiling, which was established in Breda and Santos (2004).

Proposition 5.5 Let $\tau \in \Omega(Q, T)$, such that $\tau$ has two cells in adjacent positions as illustrated in Fig. 9. $D$, then $\tau$ has six cells in adjacent positions as illustrated in Fig. 18 


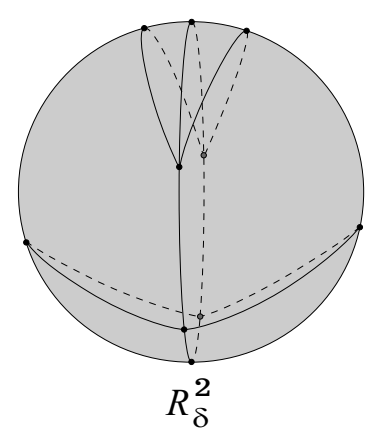

Fig. 17: 3D representation.

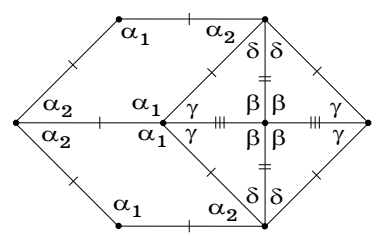

Fig. 18: Planar representation.

Proposition 5.6 If the PR illustrated in Fig. 18 is contained in a complete PR of an element $\tau \in \Omega(Q, T)$, then:

i) If $\alpha_{2} \geq \frac{\pi}{2}$, then $\alpha_{1}=\frac{2 \pi}{3}, \alpha_{2}=\frac{\pi}{2}=\beta, \gamma=\frac{\pi}{3}$ and $\delta=\frac{\pi}{4}$;

ii) If $\alpha_{2}<\frac{\pi}{2}$, then $\alpha_{1}=\frac{2 \pi}{3}, \alpha_{2}=\frac{2 \pi}{5}, \beta=\frac{\pi}{2}, \gamma=\frac{\pi}{3}$ and $\delta=\frac{\pi}{5}$.

Moreover, the first situation leads to two dihedral f-tilings denoted by $M^{3}$ and $S^{2}$, and in the second case one gets three dihedral f-tilings denoted by $G_{1}, G_{2}$ and $G_{3}$, respectively. In any case $Q$ is decomposed in four triangles congruent to $T$, bisecting $Q$ twice, by $\alpha_{1}$ and by $\alpha_{2}$. The sum of alternating angles around vertices are represented in Fig. 19] For a 3D representation of the mentioned f-tilings, see Fig. 20

Finally we present the f-tilings obtained from the adjacency illustrated in Fig. 9-E or Fig. 9-F.

Proposition 5.7 Let $\tau$ be an element of $\Omega(Q, T)$, such that $\tau$ has two cells in adjacent positions as illustrated in Fig. 9. E or Fig. 9r.F, then

$$
\left.\tau=R_{\phi}^{k}, \text { for some } \phi \in\right] 0, \arccos \frac{1-\cos \frac{\pi}{k}}{2}[\backslash\{\pi / k\} \text { and } k \geq 3 .
$$

Any planar representation of $R_{\phi}^{k}$ has the configuration of Fig. 5 (replacing one of the $\gamma$ angles by $\delta$ ) and the sum of alternating angles around vertices are given by:

i) $\alpha_{1}+\delta=\pi, \alpha_{2}+\beta=\pi\left(\beta>\alpha_{2}\right)$ and $\gamma=\frac{\pi}{k}, k \geq 3$, when considered the adjacency illustrated in Fig. 9r. E. In this case $\phi=\delta \in] 0, \frac{\pi}{k}[$; 


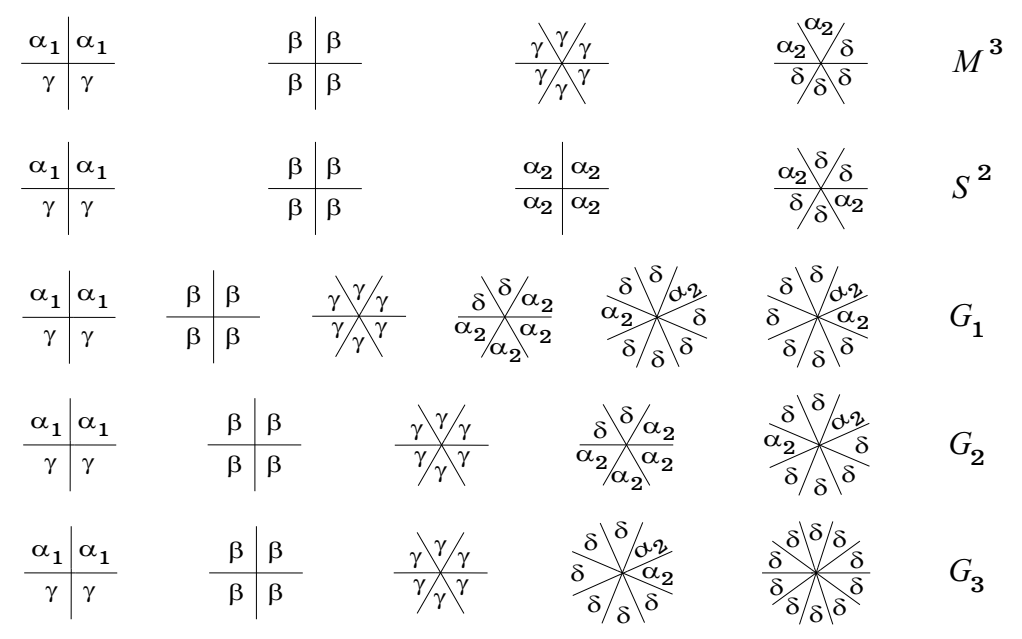

Fig. 19: Distinct classes of congruent vertices.
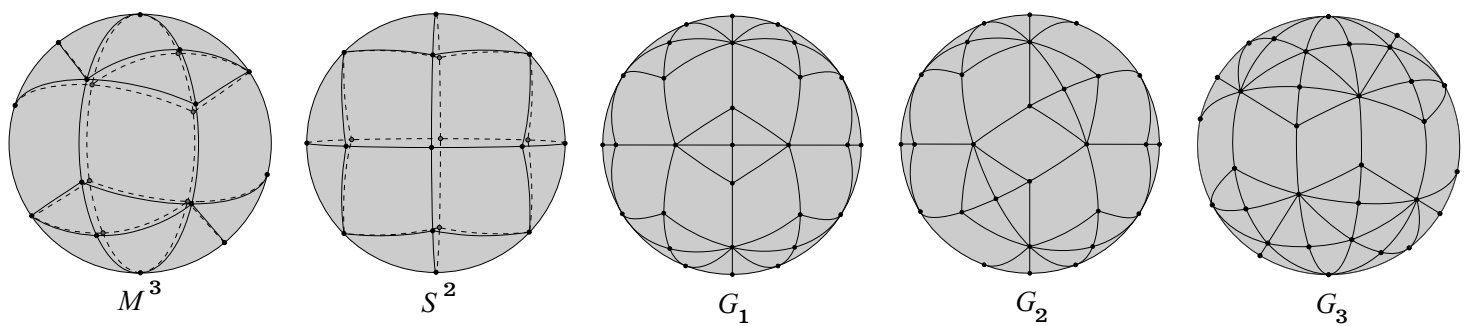

Fig. 20: 3D representations. 
or

ii) $\alpha_{1}+\gamma=\pi, \alpha_{2}+\beta=\pi$ and $\delta=\frac{\pi}{k}, k \geq 3$, when considered the adjacency illustrated in Fig. 9F. Here $\phi=\gamma \in] \frac{\pi}{k}, \arccos \frac{1-\cos \frac{\pi}{k}}{2}[$.

In both cases $\cos \beta+\cos \gamma+\cos \delta=1$.

Proof: We shall suppose that $\tau \in \Omega(Q, T)$ has two cells in adjacent positions as illustrated in Fig. 9.E (the other case is analogous). Using similar argumentation to the one used before we obtain in a unique way a planar representation with the configuration of Fig. 5 (replacing one of the $\gamma$ angles by $\delta$ ), where $\alpha_{1}+\delta=\pi, \alpha_{2}+\beta=\pi$ and $\gamma=\frac{\pi}{k}$, for some $k \geq 3$.

Now, as $Q$ has all congruent sides and distinct pairs of opposite internal angles, then $Q$ is congruent to a WCSQ, as seen in Breda and Santos (2003). This means that $Q=L_{1} \cap L_{2}$, for some non-orthogonal well centered spherical moons $L_{1}$ and $L_{2}$, with angle measure $\gamma=\frac{\pi}{k}(k \geq 3)$, see Fig. 21 .

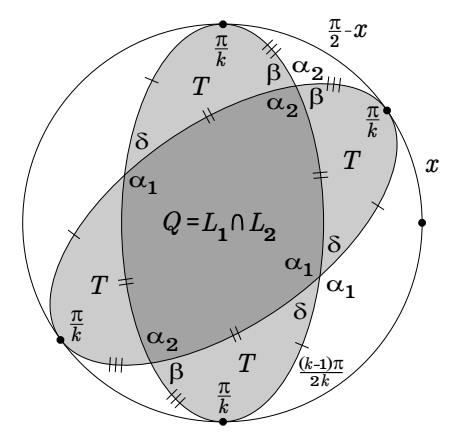

Fig. 21: A rhombus, $Q=L_{1} \cap L_{2}$.

With the labelling of Fig. 21, one gets

$$
\cos \beta=\frac{1-\cos \frac{\pi}{k}}{2}-\frac{1+\cos \frac{\pi}{k}}{2} \sin x
$$

and

$$
\cos \delta=\frac{1-\cos \frac{\pi}{k}}{2}+\frac{1+\cos \frac{\pi}{k}}{2} \sin x,
$$

for some $x \in] 0, \frac{\pi}{2}\left[\right.$ and $k \geq 3$. And so $\cos \frac{\pi}{k}+\cos \beta+\cos \delta=1$.

The relations (5.4) and (5.5) define, respectively, an increasing continuous bijection between $x \in] 0, \frac{\pi}{2}$ [ and $\beta \in] \arccos \frac{1-\cos \frac{\pi}{k}}{2}, \frac{(k-1) \pi}{k}[$, and a decreasing continuous bijection between $x \in] 0, \frac{\pi}{2}[$ and $\delta \in$ ] $0, \arccos \frac{1-\cos \frac{\pi}{k}}{2}[$.

Note that $\gamma \leq \frac{\pi}{3}<\arccos \frac{1-\cos \frac{\pi}{k}}{2}<\frac{\pi}{2}$, for all $k \geq 3$. Now, as $\beta>\gamma=\frac{\pi}{k}>\delta$, then exists $\left.x_{k} \in\right] 0, \frac{\pi}{2}[$, such that $0<\delta=\delta(x)<\frac{\pi}{k}$, for all $\left.x \in\right] x_{k}, \frac{\pi}{2}[$ and $k \geq 3$. In fact,

$$
\delta<\frac{\pi}{k} \Leftrightarrow \cos \delta(x)>\cos \frac{\pi}{k} \Leftrightarrow \sin x>\frac{-1+3 \cos \frac{\pi}{k}}{1+\cos \frac{\pi}{k}} .
$$


And so

$$
x_{k}=\arcsin \left(3-\frac{4}{1+\cos \frac{\pi}{k}}\right) .
$$

Taking $\beta_{k}=\beta\left(x_{k}\right)$ (i.e., $\left.\delta=\frac{\pi}{k}\right)$, then one gets $\beta_{k}=2 \arccos \sqrt{1-\cos \frac{\pi}{k}}=\arccos \left(1-2 \cos \frac{\pi}{k}\right)$.

One has $\beta_{3}=\frac{\pi}{2}$ and $\frac{\pi}{2}<\beta_{k}<\pi$, if $k \geq 4$. And so $\beta=\beta(x)>\beta_{k} \geq \frac{\pi}{2}>\alpha_{2}$, for all $\left.x \in\right] x_{k}, \frac{\pi}{2}[$ and $k \geq 3$.

The graphic representation of $\beta$ and $\delta$ is shown in Fig. 22

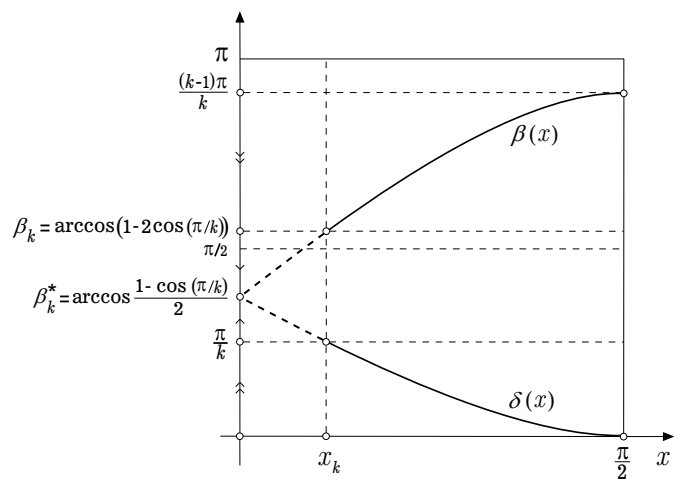

Fig. 22: Graphic representation of $\beta$ and $\delta$.

In Fig. 23 are illustrated 3D representations of $R_{\phi}^{3}$ and $R_{\phi}^{4}$.

Remark: In the ii) case of Prop. 5.7 $x$ runs the interval $] 0, x_{k}[. \beta$ and $\gamma$ are determined by $x$. Besides, considering the limit case $x=0$, then $\gamma=\beta=\beta_{k}^{*}=\arccos \frac{1-\cos \frac{\pi}{k}}{2}$ and so $\alpha_{1}=\alpha_{2}$, giving rise to a dihedral f-tiling established in Breda and Santos (2004). The case $x=x_{k}(k \geq 3)$ leads to the f-tiling $R^{k}$ obtained in Prop.4.2 (see 4.1).

In Tab. 1 it is shown a complete list of all dihedral f-tilings, whose prototiles are a spherical triangle $T$ of angles $\beta, \gamma, \delta(\beta>\gamma>\delta)$ if $T$ is scalene, or angles $\beta, \gamma, \gamma(\beta \neq \gamma)$ if $T$ is isosceles, and, a non-equiangular spherical rhombus $Q$ with pairs of opposite internal angles $\alpha_{1}$ and $\alpha_{2}\left(\alpha_{1}>\alpha_{2}\right)$. We have used the following notation:

- $\beta_{k}=\arccos \left(1-2 \cos \frac{\pi}{k}\right), \beta_{k}^{*}=\arccos \frac{1-\cos \frac{\pi}{k}}{2}$, and $\delta_{k}=\arccos \left(\cos ^{2} \frac{\pi}{2 k}\right)$;

- At f-tilings $R_{\delta}^{2}, R_{\phi}^{k}(\phi=\delta$ or $\phi=\gamma)$ one has $\cos \beta+\cos \gamma+\cos \delta=1$;

- $|V|$ is the number of distinct classes of congruent vertices (we shall say that vertices $v_{1}$ and $v_{2}$ are congruent if and only if there is a spherical isometry that maps the faces incidents to $v_{1}$ into the faces incidents to $v_{2}$ );

- $M$ and $N$ are, respectively, the number of quadrangles congruent to $Q$, and the number of triangles congruent to $T$, used in such dihedral f-tilings. 

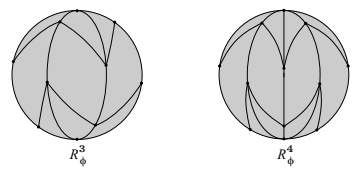

Fig. 23: 3D representations. 


\begin{tabular}{|c|c|c|c|c|c|c|c|c|}
\hline f-tiling & $\alpha_{1}$ & $\alpha_{2}$ & $\beta$ & $\gamma$ & $\delta$ & $|V|$ & $M$ & $N$ \\
\hline \hline$U_{1}$ & $\frac{2 \pi}{3}$ & $\frac{\pi}{2}$ & $\frac{\pi}{2}$ & $\frac{\pi}{3}$ & - & 5 & 6 & 12 \\
\hline$U_{2}, U_{4}$ & $\frac{2 \pi}{3}$ & $\frac{\pi}{2}$ & $\frac{\pi}{2}$ & $\frac{\pi}{3}$ & - & 3 & 8 & 8 \\
\hline$U_{3}$ & $\frac{2 \pi}{3}$ & $\frac{\pi}{2}$ & $\frac{\pi}{2}$ & $\frac{\pi}{3}$ & - & 5 & 4 & 16 \\
\hline$R^{k}, k \geq 3$ & $\frac{(k-1) \pi}{k}$ & $\pi-\beta_{k}$ & $\beta_{k}$ & $\frac{\pi}{k}$ & - & 3 & $2 k$ & $4 k$ \\
\hline$R_{\delta}^{2}$ & $\pi-\delta$ & $\pi-\gamma$ & $\frac{\pi}{2}$ & ]$\frac{\pi}{3}, \frac{\pi}{2}[$ & ] $0, \frac{\pi}{3}[$ & 3 & 4 & 8 \\
\hline$R_{\delta}^{k}, k \geq 3$ & $\pi-\delta$ & $\pi-\beta$ & ]$\beta_{k}, \frac{(k-1) \pi}{k}[$ & $\frac{\pi}{k}$ & ] $0, \frac{\pi}{k}[$ & 3 & $2 k$ & $4 k$ \\
\hline$R_{\gamma}^{k}, k \geq 3$ & $\pi-\gamma$ & $\pi-\beta$ & ]$\beta_{k}^{*}, \beta_{k}[$ & ]$\frac{\pi}{k}, \beta_{k}^{*}[$ & $\frac{\pi}{k}$ & 3 & $2 k$ & $4 k$ \\
\hline$M^{3}$ & $\frac{2 \pi}{3}$ & $\frac{\pi}{2}$ & $\frac{\pi}{2}$ & $\frac{\pi}{3}$ & $\frac{\pi}{4}$ & 4 & 6 & 24 \\
\hline$M^{k}, k \geq 4$ & $\frac{(k-1) \pi}{k}$ & $\pi-2 \gamma$ & $\frac{\pi}{2}$ & $\frac{\beta_{k}}{2}$ & $\frac{\pi}{k}$ & 4 & $2 k$ & $8 k$ \\
\hline$E^{k}, k \geq 3$ & $\pi-\delta$ & $\frac{\pi}{2 k-3}$ & $\frac{\pi}{2}$ & $\frac{(k-2) \pi}{2 k-3}$ & $\delta_{2 k-3}$ & 4 & $8 k-12$ & $16 k-24$ \\
\hline$S^{2}$ & $\frac{2 \pi}{3}$ & $\frac{\pi}{2}$ & $\frac{\pi}{2}$ & $\frac{\pi}{3}$ & $\frac{\pi}{4}$ & 4 & 8 & 16 \\
\hline$S^{k}, k \geq 3$ & $\pi-\delta$ & $\frac{\pi}{k}$ & $\frac{\pi}{2}$ & $\frac{(k-1) \pi}{2 k}$ & $\delta_{k}$ & 4 & $4 k$ & $8 k$ \\
\hline$G_{1}$ & $\frac{2 \pi}{3}$ & $\frac{2 \pi}{5}$ & $\frac{\pi}{2}$ & $\frac{\pi}{3}$ & $\frac{\pi}{5}$ & 6 & 16 & 56 \\
\hline$G_{2}$ & $\frac{2 \pi}{3}$ & $\frac{2 \pi}{5}$ & $\frac{\pi}{2}$ & $\frac{\pi}{3}$ & $\frac{\pi}{5}$ & 5 & 18 & 48 \\
\hline$G_{3}$ & $\frac{2 \pi}{3}$ & $\frac{2 \pi}{5}$ & $\frac{\pi}{2}$ & $\frac{\pi}{3}$ & $\frac{\pi}{5}$ & 5 & 10 & 80 \\
\hline
\end{tabular}

Tab. 1: Dihedral spherical f-tilings by triangles and non-equiangular rhombi.

In Fig. 24 it is shown a 3D representation of the dihedral f-tilings described on Tab. 1 Observe that for the families $M^{k}, E^{k}$ and $S^{k-1}, k \geq 3$ we have chosen $M^{5}, E^{3}$ and $S^{3}$ as representatives for their 3D representations.

\section{Acknowledgements}

The authors are grateful to the referees for their helpful comments and improvements. 
Fig. 24: 3D representations of the dihedral spherical f-tilings by triangles and non-equiangular rhombi. 


\section{References}

M. Berger. Geometry, volume 2. Springer-Verlag, New York, 1996.

A. M. Breda. A class of tilings of $s^{2}$. Geometriae Dedicata, 44(3):241-253, 1992.

A. M. Breda and A. F. Santos. Well centered spherical quadrangles. Beiträge zur Algebra und Geometrie / Contributions to Algebra and Geometry, 44(2):339-349, 2003. available at http://www.emis.de/journals/BAG/vol.44/no.2/22.html.

A. M. Breda and A. F. Santos. Dihedral f-tilings of the sphere by spherical triangles and equiangular well-centered quadrangles. Beiträge zur Algebra und Geometrie / Contributions to Algebra and Geometry, 45(2):447-461, 2004. available at http://www.emis.de/journals/BAG/vol.45/no.2/8.html.

H. L. Davies. Packings of spherical triangles and tetrahedra. Proccedings of the Colloquium on Convexity (ed. W. Fenchel), K. Univ. Mat. Inst., Copenhagen, pages 42-51, 1967.

B. Grünbaum and G. C. Shephard. Tilings and Patterns. W. H. Freeman and Company, 1986.

S. A. Robertson. Isometric foldings of riemannian manifolds. Proc. R. Soc. Edinb., Sect. A, 79:275-284, 1977.

D. Sommerville. Division of space by congruent triangles and tetrahedra. Proc. Royal Soc. Edinb., 43(3): 85-116, 1922.

Y. Ueno and Y. Agaoka. Classification of tilings of the 2-dimensional sphere by congruent triangles. Hiroshima Math. Journal, 32(3):463-540, 2002. 
